\title{
Practical Channel Measurements and Models of 3-D Multi-User Distributed Massive MIMO Systems
}

\author{
Aghaei Nezhad Firouzja $\mathbf{S}^{1 *}$ and Asghari Hemmat Abadi $\mathbf{M}^{2}$ \\ ${ }^{1}$ Department of Electronics Engineering, Shanghai Jiao Tong University, Shanghai, P.R. China \\ ${ }^{2}$ Department of Mathematics, Guangzhou University, Guangzhou, P.R. China
}

\begin{abstract}
This paper presents a practical three-dimensional (3-D) outdoor channel model for multi-user distributed massive multi-input multi-output (MU-DMMIMO). As the dimension of the antenna and the distance between transmitter and receiver increasing, the elevation angles will be more challenge, especially at the cell edge. In this paper, the beam pattern is assumed in the proposed channel model instead of the spherical wave front assumption used in current 3-D channel models. These include the far-field effects were done jointly with azimuth and elevation angles in different appearance clusters with various times of elevation and azimuth angle of departure and arrival on both the time and array axes. In addition, their impacts on MU-DMMIMO at the cell edge are investigated via statistical properties including a measurement of the path-loss power control on the non-stationary model of the user at the receiver. It is demonstrated that the proposed 3-D channel model is more accurate in characterizing real propagation channel when users are handovers at the cell edge.
\end{abstract}

\section{Keywords: 3-D; MU-DMMIMO; Path-loss}

\section{Introduction}

MULTI user multi-input multi-output (MU-MIMO) channel modeling is a fundamental step that provides a performance evaluation of the transceiver technique. Prior to this, 3-D channel models has attracted substantial research attention as an important technique to improve vertical coverage and overall system capacity thus, providing additional degrees of freedom to meet the high level of demand. In current standardized channel models, all the user equipment's (UEs) are assumed to have a height of $1.5 \mathrm{~m}$. Prior to this, the elevation angles depend on the distance between the base station (BS) and the UE. Therefore, the elevation angle of departure (EAoD) and elevation angle of arrival (EAoA) specifically are the new parameters of the 3-D channel model to improve the vertical angle, especially in the far-field condition.

To design and evaluate the massive MIMO systems, it is necessary to have an accurate and efficient massive MIMO channel models. In parallel to this work, there has been a potential interest to develop the framework of wireless standards, reference channel models which serve to define conventional ways to generate channels [1]. Many researchers investigated the channel modeling with considering the antenna configuration and polarization. In [2], a close form expression of the spatial correlation as a function of the physical parameters representing characteristics of 3-D MIMO derived. In [3] presented a generalized MIMO channel modeling technique combining the spatial correlation and wave position in the direction of antenna arrays to achieve both accuracy and efficiency. In [4] investigated the impact of elevation angles for a MIMO system with a small number of antennas. In [5], the model may analyze quantitatively the parameters which can affect the channel capacity-antenna pattern for an optimal antenna structure for MIMO systems. In [6], the paper addresses the extension of a stochastic geometry-based scattering model to multi-polarized transmissions to investigate various dual-polarizations for MIMO antenna system. In $[7,8]$, the work was based on the 3-D theoretical channel model. The authors assumed an infinite number of effective scatters with resulting infinite complexity that can hardly be put into practical use.

However, according to measurement observation in [9-11], the above-mentioned channel modeling are not sufficient to accurately capture certain characteristics of 3-D multi-user distributed massive MIMO channels in the far-field condition. For the sake of massive MIMO experimental design, we propose 3-D beam pattern channel models to detect the characteristics of the elevation and azimuth angle accurately with a higher degree of freedom. All the measurement results are obtained from mathematical derivation and practical work at Shanghai Jiao Tong University (SJTU) campus network as shown in Figure 1. Our main contributions are : 1) The impact of beam patterns has been proposed to providing different phase excitation, contributes various AoDs and AoAs for rays related towards/from the clusters. 2) Far-field effects caused by investigation user handovers at the cell edge based on the distance between a serving cell and UE is investigated using reference signal received power (RSRP) and proposed pathloss channel model, and later prior to this, the position of UE is more accurately estimate using the azimuth and elevation angles, including beam pattern of the serving cell. 3) Statistical properties of the proposed channel model such as path-loss measurements considering RSRP, reference signal receive quality (RSRQ) and received signal strength indicator (RSSI), including signal-to-interference-plus-noise ratio (SINR) of the non-stationary channel model are investigated. This leads us to accurately classify the user's complicated relation to its own and neighboring cells in order to keep the signal quality constant. As far as the author's knowledge is concerned, this is the first practical 3-D multiuser distributed massive MIMO channel model using beam pattern wave front for the far-field effects.

*Corresponding author: Aghaei Nezhad Firouzja S, Department of Electronics Engineering, Shanghai Jiao Tong University, Shanghai, P.R. China, Tel:+86 13020208949; E-mail: fs.aghaeig@sjtu.edu.cn

Received October 10, 2018; Accepted October 31, 2018; Published November 07, 2018

Citation: Firouzja SAN, Abadi MAH (2018) Practical Channel Measurements and Models of 3-D Multi-User Distributed Massive MIMO Systems. J Electr Electron Syst 7: 280. doi: 10.4172/2332-0796.1000280

Copyright: ( 2018 Firouzja SAN, et al. This is an open-access article distributed under the terms of the Creative Commons Attribution License, which permits unrestricted use, distribution, and reproduction in any medium, provided the original author and source are credited. 
Citation: Firouzja SAN, Abadi MAH (2018) Practical Channel Measurements and Models of 3-D Multi-User Distributed Massive MIMO Systems. J Electr Electron Syst 7: 280. doi: 10.4172/2332-0796.1000280

\section{3-D Distributed Massive Mimo Channel Modeling With Practical Beam Patterns}

\section{Massive MIMO channel}

In this section, a 3-D channel model for Distributed MU-massive MIMO is proposed, considering beam pattern to improve the elevation angles for the far-field effects. The models are defined by two effective physical objectives, one around the transmitter (TX), and other one is around the receiver (RX). As shown in the Figure 2, two spherical models for dipole antennas and Omni-directional antennas to minimize the clusters dispersing both for TX and RX, respectively. Let us now consider $\mathrm{L} \times \mathrm{K}$ pairs of transmit antenna elements indexed by $(l k)_{t h}$, where $l_{t h}$ row with $\mathrm{l}=(0, \ldots, \mathrm{L}-1)$ and $k_{t h}$ column with $k=(0, \ldots$, $K-1)$ and similarity, $L^{\prime} \times K^{\prime}$ pairs of receive antenna elements indexed by $\left(l^{\prime}, k^{\prime}\right)_{t h}$, where $l_{\text {th }}^{\prime}$ row with $l^{\prime}=\left(0, \ldots ., L^{\prime}-1\right)$ and $k_{t h}^{\prime}$ column with $k^{\prime}$ $=\left(0, \ldots K^{\prime}-1\right)$. Denote $\left(\theta_{E A O D}, \varphi_{A A O D}\right)$ and $\left(\vartheta_{E A O A}, \phi_{A A O A}\right)$ as the elevation and the azimuth angles at the TX and the RX, respectively. In addition, the wave direction is fixed along $y$ axis and the massive MIMO antenna arrays are fixed at $x-z$ axes. $(\mathrm{L}, \mathrm{K})$ is a point of $(x, y, z)$ of the distance above the origin $\mathrm{O}$ from an array at the $(x, z)$ axes, where the location of first TX antenna element is indexed by $(0,0)_{\text {th }}$ and the location of the $(0,0)_{\text {th }}$ receive antenna element is $\left\{x_{(0 ; 0)}, \mathrm{y}_{(0 ; 0)}, \mathrm{z}_{(0 ; 0)}\right\}$.

For this purpose, some important aspects to characterize the outdoor massive MIMO for the far-field effects are need to be investigated, such as the distance between TX and RX, cluster generation/appearance on the antenna array, movement direction of clusters and movement direction of antenna arrays from cell to cell. Figure 2 shows all the details and dimensions of the non-stationary communication scenario for non-line-of-sight (NLOS) components. In the current setting, the testbed operates with multiple parameters as shown in Table 1. However, to generate the channel coefficient, two parts must be modeled from the transmitter toward the receiver such as a) toward the outdoor cluster, and b) toward the receive antenna elements, respectively.

Toward the outdoor cluster: Let us denote the $q t=\{1, \ldots, \mathrm{Qt}\}$, where Qt is equal to Cluster ${ }_{t}=\mathrm{Qt}(\mathrm{t}+\Delta \mathrm{t})$ as total clusters, in which $i_{1}^{\prime}$ th path from (LK) antenna elements are add up with time $(\mathrm{t}+\Delta \mathrm{t})$. In prior of this, the first path from $(0,0)_{t h}$ antenna element with time $\mathrm{t}$ is add up to the nearest cluster. And then, the rest of the paths from $(l k)_{t h}$ adjacent element calculating with distance $d^{B S}$ are add up with succeeding time instantaneously $(\Delta \mathrm{t})$ to the same cluster.

Toward the receive antenna elements: $q t_{t h}$ cluster will be observed to the $q r_{t h}$ cluster at the $\mathrm{RX}$, where $\mathrm{qr}=\{1, \ldots, \mathrm{Qr}\}$, where $\mathrm{Q} r$ is equal to Cluster ${ }_{t}=Q r(t+\Delta t)$ as total clusters, in which arrive to the $\left(\mathrm{L}^{\prime}, \mathrm{K}^{\prime}\right)$ ${ }_{t h}$ antenna element with time $(\mathrm{t}+\Delta \mathrm{t})$. Similarly, the first cluster with time $t$ is arrived to the $(0,0)_{t h}$ antenna element via $i_{2 t h}^{\prime}$ path. And then, the rest of the clusters are arriving to adjacent element with succeeding time instantaneously $(\Delta \mathrm{t})$ calculating with distance $d^{U E}$. As an example, cluster $q t 1$ is observed to the qr1 cluster, where qr1 is observed to at the $l^{\prime}, \mathrm{k}^{\prime}$ antenna elements, where first cluster is arriving at $(0,0)_{t h}$ antenna element. Therefore, the total distances between the transmit antenna array and the receive antenna array can be calculated by $\mathrm{D}=(\mathrm{D}, 0,0)$. However, to perform the evolution of the outdoor channel coefficient the total clusters from different cells toward to RX can be presented as Table 1.

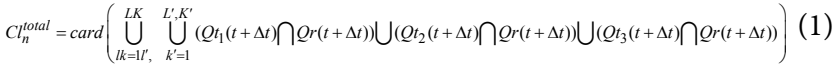

where $\mathrm{Q} t_{1}(t+\Delta t), \mathrm{Q} t_{2}(t+\Delta t)$ and $\mathrm{Q} t_{3}(t+\Delta t)$ are the total generated clusters at the cell one, two and three, respectively. In Equation (1) the total number of clusters from three cells is presented, because of the page limited, we have simplified the system by explaining the channel coefficient for a single cell. Based on the outdoor model, the multipath gain between the transmit antenna array and the receive antenna array will be denoted as $C l_{n}^{\text {out }}$ is the total number of clusters, where $\mathrm{n}=1, \ldots$, $\mathrm{N}$ cluster for outdoor communication for $\mathrm{Q} t_{1}(t+\Delta t)$ can be expressed as

$$
C l_{n}^{\text {out }}=\operatorname{card}\left(\bigcup_{l k=1 l^{\prime},}^{L K} \bigcup_{k^{\prime}=1}^{L^{\prime}, K^{\prime}}(Q t(t+\Delta t) \bigcap(Q t(t+\Delta t)))\right.
$$

where the operator card (.) denotes the cardinality of a set $[12,13]$. The operator card $C l_{n}^{\text {out }}$, each cluster, say $\operatorname{Cluster}_{n}\left(n=1, \ldots, C l_{n}^{\text {out }}\right)$ is for outdoor channel models. Based on the above analysis and the summary of key parameter definitions in Table 1, the massive MIMO channel matrix can be expressed as an $\mathrm{LK} \times \mathrm{L}^{\prime}, \mathrm{K}^{\prime}$ complex matrix $\boldsymbol{H}\left(\mathrm{t}^{\prime}, \Delta \mathrm{t}, \tau\right)=$ $\mathrm{h}_{l k, l^{\prime}, \mathrm{k}^{\prime}}(t+t, \tau)_{L K \times L^{\prime}, \mathrm{K}}{ }^{\prime}$. The amplitude complex gains between the $(l k)_{t h}$ antenna element at the LK transmit antenna, $\left(\mathrm{l}^{\prime}, \mathrm{k}^{\prime}\right)_{t h}$ antenna element at the $\mathrm{L}^{\prime}, \mathrm{K}^{\prime}$ receive antenna and the delay, can be represented as

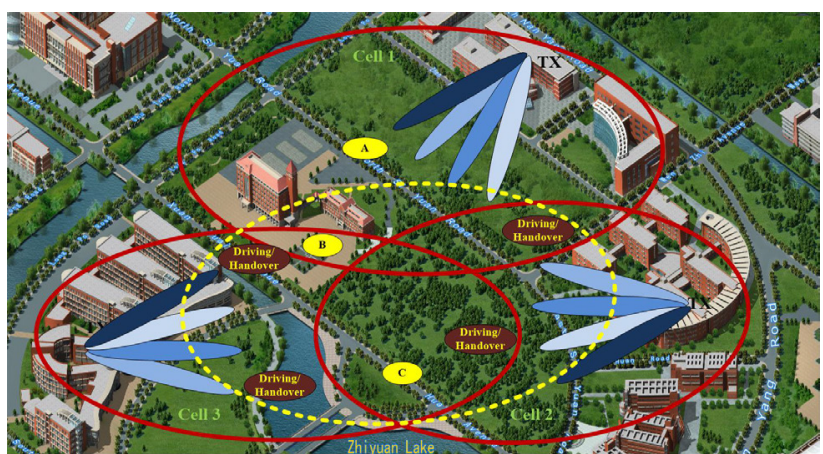

Figure 1: Multi-user Distributed Massive MIMO transceiver model system.

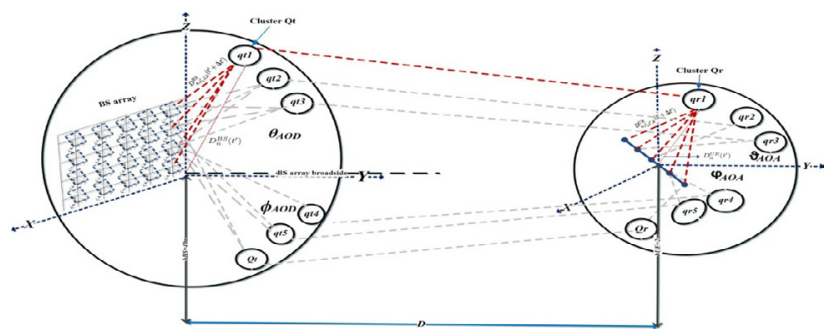

Figure 2: A more detailed of field experiments of the beam pattern

\begin{tabular}{|c|c|}
\hline Parameters & Values \\
\hline$(\theta, \varphi),(\vartheta, \phi)$ & $\begin{array}{c}\text { elevation and azimuth angle of the departures and arrivals, } \\
\text { respectively }\end{array}$ \\
\hline$\left.D_{n, i_{1}^{\prime}, l k}^{B S}(t+\Delta t)\right]$ & $\begin{array}{c}\text { distance vector between } n_{t h} \text { cluster and }(I k)_{t h} \text { transmit antenna } \\
\text { element via } i_{1 t h}^{\prime} \text { path }\end{array}$ \\
\hline$D_{n, i_{1}^{\prime},}^{B S}(t)$ & distance vector between $n_{t h}$ cluster and transmit antenna via $i_{1 t h}^{\prime}$ path \\
\hline$D_{n, i_{2}, l^{\prime}, k^{\prime}}^{U E}\left(t^{\prime}\right)$ & $\begin{array}{c}\text { distance vector between } n_{\text {th }} \text { cluster and }\left(l^{\prime}, k^{\prime}\right)_{t h} \text { receive antenna } \\
\text { element via } i_{2 t h}^{\prime} \text { path }\end{array}$ \\
\hline$D_{n, i_{2}^{2},}^{U E}\left(t^{\prime}\right)$ & distance vector between $n_{t h}$ cluster and receive antenna via ${ }^{i_{2 t h}^{\prime}}$ path \\
\hline $\begin{array}{l}{\left[\vec{a}(\phi, \theta]_{l k}\right.} \\
{[\vec{a}(\varphi, \vartheta)]_{l^{\prime}, k^{\prime}}}\end{array}$ & array response of the $\mathrm{TX}(\mathrm{X}-\mathrm{Pol})$ and $\mathrm{RX}(\mathrm{V}-\mathrm{Pol})$ polarization \\
\hline
\end{tabular}
coordinate system for distributed massive MIMO channel.

Table 1: Summary of Key Parameter Definitions. 
Citation: Firouzja SAN, Abadi MAH (2018) Practical Channel Measurements and Models of 3-D Multi-User Distributed Massive MIMO Systems. J Electr Electron Syst 7: 280. doi: 10.4172/2332-0796.1000280

Page 3 of 4

$h_{l k, l^{\prime}, k^{\prime}}(t+\Delta t, \tau)=\sum_{n=1}^{C l_{n}^{\text {out }}} h_{n, l k, l^{\prime}, k^{\prime}}(t+\Delta t) \delta\left(\tau-\tau_{n}\right)$

If Clusters

$\in \bigcup_{l k=1}^{L K} \bigcup_{l^{\prime}, k^{\prime}=1}^{L^{\prime}, 1}(Q t(t+\Delta t) \bigcap Q r(t+\Delta t))$,

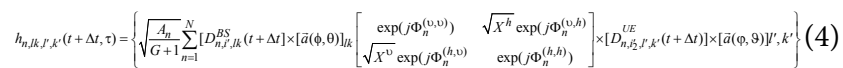

where a Cluster ${ }_{\mathrm{n}} \in\left(\mathrm{Q} t(t+t), \mathrm{Q} r(t+\Delta t) \leq C l_{n}^{\text {out }}\right.$ is observed for outdoor model. $G$ denote Rician factor, the power of the $n_{\text {th }}$ cluster is defined as $A_{n}$. The calculation of complex gains for NLOS components in Equation (4) can be presented as follow:

At the transmitter: The $(\mathrm{lk})_{t h}$ transmit antenna element vector $[\vec{a}(\phi, \theta)]_{l k}$, where $[\vec{a}(\phi, \theta)]_{l k}=e^{\left(\omega_{l k} \frac{2 \pi}{\lambda} \hat{y} \cdot d^{B S}\right)}$ and the vector between $\mathrm{n}_{t h}$ cluster via $i_{t h}^{\prime}$ path can be presented as $D_{n, i_{1}, l k}^{B S}(t+\Delta t) \cdot \omega_{l k}$ is beam weight with transmit antenna elements. The vector between the $n_{\text {th }}$ cluster and transmit antenna array is denoted by $D_{n}^{B S}\left(t^{\prime}\right)$ at the TX. $\hat{y}=[\sin \phi \cos \theta, \sin \phi \sin \theta, \cos \theta]$ is the outgoing wave direction.

$$
\left[D_{n, i_{1}^{\prime}, l k}^{B S}(t+\Delta t)\right]=D_{n}^{B S}\left(t^{\prime}\right)\left[\begin{array}{c}
\sin \phi_{n, i_{1}^{\prime}, l k}^{A A o D}(t+\Delta t), \cos \theta_{n, i_{1}^{\prime}, l k}^{E A D D}(t+\Delta t) \\
\sin \theta_{n, i_{1}^{\prime}, l k}^{A A O D}(t+\Delta t), \sin \theta_{n, i_{1}^{\prime}, k}^{E A o D}(t+\Delta t) \cos \theta_{n, i_{1}^{\prime}, l k}^{E A o D}(t+\Delta t)
\end{array}\right]^{T}
$$

At the receiver: Similarly, the $\left(l^{\prime}, \mathrm{k}^{\prime}\right)_{t h}$ receive antenna element vector $[\vec{a}(\varphi, \vartheta)]_{l^{\prime} k^{\prime}}$ where and the vector between $\mathrm{n}_{\text {th }}$ cluster via $i_{2 t h}^{\prime}$ path can denoted as $D_{n, i_{2}, l^{\prime} k^{\prime}}^{U E}(t+\Delta t) . \hat{y}^{\prime}=[\sin \varphi \cos \vartheta, \sin \varphi \sin \vartheta, \cos \vartheta]$ for the incoming wave in the response of the antenna. The polarization angle $\alpha$ from the $z$ axis, has vertical and horizontal components of the antenna pattern of $\left[\begin{array}{c}\cos \alpha \sin \vartheta+\cos \alpha \cos \vartheta \cos \varphi \\ \sin \alpha \cos \phi\end{array}\right]$. Furthermore, the vector between the $n_{\text {th }}$ cluster and the receive antenna array is denoted by $D_{n}^{U E}\left(t^{\prime}\right)$.

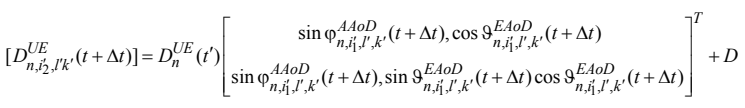

Then, the four random initial phases for $n_{\text {th }}$ cluster are derived as
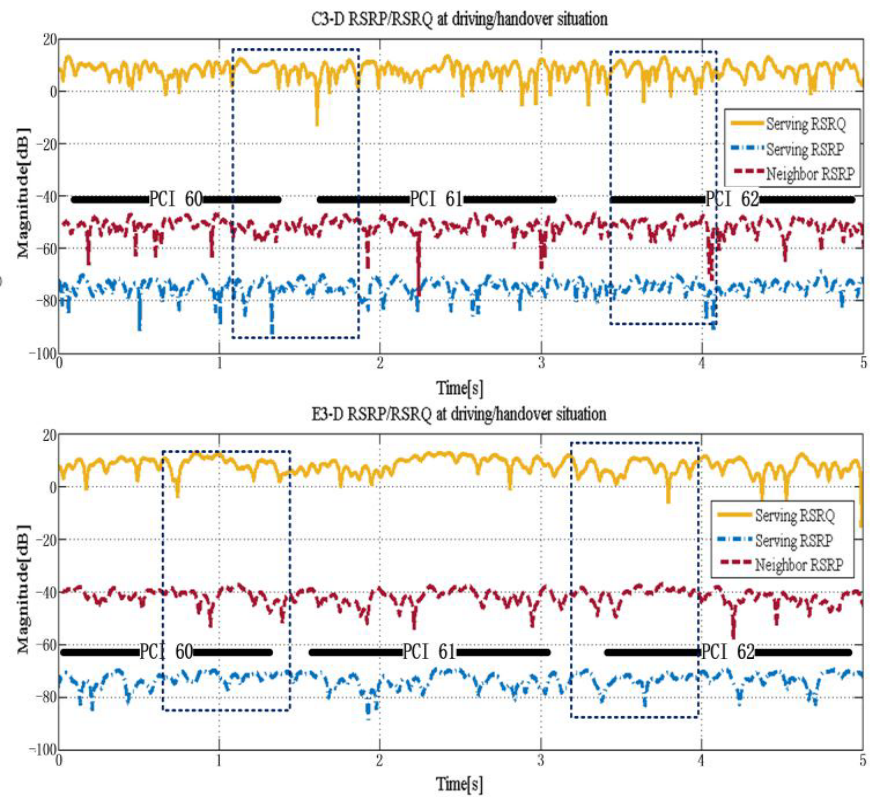

Figure 3: A RSRP/RSRQ at driving/handover of neighbor/serving cells and RSRP/RSRQ/RSSI/SINR at driving/handover situation.

Where $\sqrt{X^{h}}$ and $\sqrt{X^{v}}$ are inverse polarized for X-Pol (vv/ hv and hh/vh) transmission, respectively. $j=\sqrt{-1}$ and $\Phi_{n}^{(\mathrm{v}, \mathrm{v})}$, $\Phi_{n}^{(\mathrm{v}, h)}, \Phi_{n}^{(h, \mathrm{v})}, \Phi_{n}^{(h, h)}$ are the four random initial phases of different polarization combination.

If Clusters $\quad \notin \bigcup_{l k=1}^{L K} \bigcup_{l^{\prime}, k^{\prime}=1}^{L^{\prime}, K^{\prime}}(Q t(t+\Delta t) \bigcap Q r(t+\Delta t)), \quad$ the channel $h_{n, l k, l^{\prime}, k^{\prime}}(t+\Delta t, \tau)=0$

\section{UE Dropping}

In LTE-A network, the determination of the serving cell for each UE is based on a metric known as RSRP, only measures the power present in the time and frequency domain symbols with the reference signal and the RSRQ. Measurement provides additional information when RSRP is not sufficient in making a reliable handover or cell reselection decision. The carrier RSSI measures the received power in a received radio signal. After performing UE dropping, this phase consists in computing for each UE $\mathrm{m}$ and site $\mathrm{u}$ the following quantities [14]. The NLOS direction characterized by a spatial angles $(\varphi, \theta)$ and $(\phi, 9)$ with respected to the $u_{\text {th }}$ cell. For each cell $s$ in the site, compute the antenna gain at the TX and $\operatorname{RX~G}_{\mathrm{T}}\left(\varphi_{\mathrm{n}, \mathrm{u}, \mathrm{m}, \mathrm{s}}, \theta_{\mathrm{n}, \mathrm{u}, \mathrm{m}, \mathrm{s}}\right)$ and $\mathrm{G}_{\mathrm{R}}\left(\phi_{\mathrm{n}, \mathrm{u}, \mathrm{m},}\right.$ $\left.{ }_{s}, \vartheta_{\mathrm{n}, \mathrm{u}, \mathrm{m}, \mathrm{s}}\right)$ in $\mathrm{dB}$. Compute the path-loss $P L_{u, m}$ and shadow-fading $\sigma_{u, m}$ between the UE and cells. Define for the user, the $R S R P_{u, m, s}$

$$
\begin{aligned}
& R S R P_{u, m, s}=P_{T X}+G_{T}\left(\varphi_{n, u, m, s}, \vartheta_{n, u, m, s}\right)+ \\
& G_{R}\left(\varphi_{n, u, m, s}, \vartheta_{n, u, m, s}\right)-P L_{u, m}(d B)-\sigma_{u, m(d B)}
\end{aligned}
$$

wher $\mathrm{P}_{\mathrm{TX}}$ is the transmitted power $\mathrm{dB}$. Determine the index $\bar{u}_{m}$ the coupling gain for the UE $\mathrm{m}$ is defined as and $\bar{s}_{m}$ of the best serving cell which maximizes the RSRP.

$$
C L_{m}=R S R P_{\bar{u}_{m}, \bar{s}_{m}}-P_{T X}
$$

\section{System Measurements}

Three cells with Physical Cell ID (PCI), where PCI $=(60,61,62)$ were

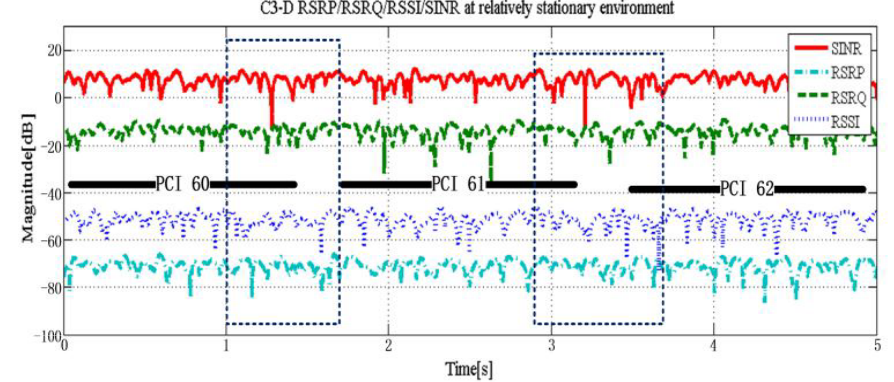

E3-D RSRP/RSRQ/RSSI/SDNR at relatively stationary environment

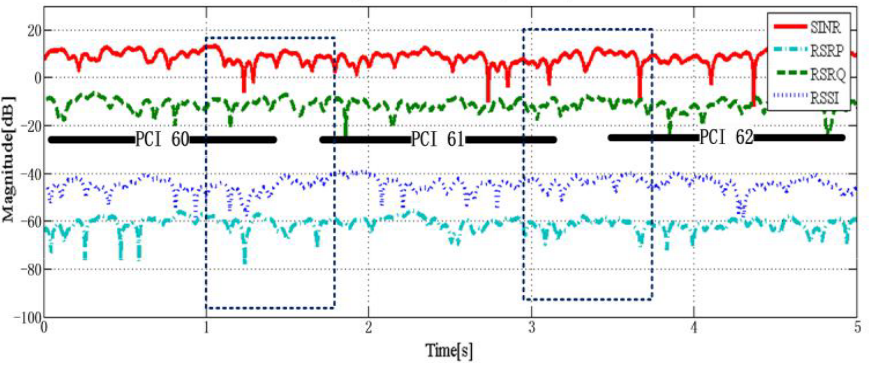


Citation: Firouzja SAN, Abadi MAH (2018) Practical Channel Measurements and Models of 3-D Multi-User Distributed Massive MIMO Systems. J Electr Electron Syst 7: 280. doi: 10.4172/2332-0796.1000280

Page 4 of 4

employed with massive MIMO antenna systems, where multiple users are randomly distributed at the cell edge as shown in Figure 1. To get a more quantitative understanding of how our proposed model would perform in the real propagation, we turned our attention to the RSRP, RSRQ, and RSSI in the two measured scenarios. The compression has been made of current channel model and proposed model denote C3-D and E3-D, respectively. In Figure 3, the difference between RSRP, RSRQ of UE (B) and RSRP, RSRQ, RSSI, SINR for UE (C) has been investigated, respectively. The rectangular dots on Figure 3 shows the RSRP and RSRQ of the neighbor/serving cells and RSRP, RSRQ, RSSI and SINR of the neighbor/serving cells at the driving/handover situations. It is illustrated that the RSRP changes pretty dynamically even along the straight open space. In addition, many changes PCI of the serving cell, meaning the UE has gone through many Handovers and at most of the places where handover happens because there is a certain point where neighbor cell RSRP is higher than the serving cell RSRP. Based on the situation, all of these values drop at the beginning and increase around the end of this section. It is also illustrated, all of the measurement faded suddenly cusecs two possibilities what call drop or UE switched to other RAN and later all the measurement gaining again. However, the result confirmed the potential of elevated 3 -D beam pattern in the enhancement of system performance.

\section{Conclusion}

An investigation has been conducted considering the effect of the received signal quality from different reference signals on the performance of MU-DMMIMO system when there is handover from cell to cell communication. It has been shown that our model for MU-DMMIMO clearly improved the signal strength/quality of the neighboring cell which can be achieved through different channels. It also allows us to accurately classify the user's complicated relation to its own and neighboring cells in order to keep the signal quality constant. However, the model is covering current existing theoretical limitations to manual testing, which may miss some important measurement spots.

\section{References}

1. Nadeem QUA, Kammoun A, Debbah M, Alouini MS (2015) A generalized spatial correlation model for $3 \mathrm{~d}$ mimo channels based on the fourier coefficients of power spectrums. IEEE Trans Signal Process 63: 3671-3686.

2. Dao MT, Nguyen VA, Im YT, Park SO, Yoon G (2011) 3d polarized channe modeling and performance comparison of mimo antenna configurations with different polarizations. IEEE Trans Antennas Propag 59: 2672-2682.

3. Xu H, Chizhik D, Huang H, Valenzuela R (2004) A generalized space-time multiple-input multiple-output (mimo) channel model. IEEE Trans Wireless Commun 3: 966-975.

4. Shafi M, Zhang M, Moustakas AL, Smith PJ, Molisch AF (2006) Polarized mimo channels in 3-d: models, measurements and mutual information. IEEE $J$ Sel Areas Commun 24: 514-527.

5. Oh S, Myung N (2004) Mimo channel estimation method using ray-tracing propagation model. Electron Lett 40: 1.

6. Oestges C, Erceg V, Paulraj AJ (2004) Propagation modeling of mimo multipolarized fixed wireless channels. IEEE Trans Veh Technol 53: 644-654.

7. Yuan $Y$, Wang $C X, \mathrm{He} Y$, Alwakeel MM (2015) 3d wideband non-stationary geometry-based stochastic models for non-isotropic mimo vehicle-to-vehicle channels. IEEE Trans Wireless Commun 14: 6883-6895.

8. Renaudin O, Kolmonen VM, Vainikainen P, Oestges C (2010) Non-stationary narrowband mimo inter-vehicle channel characterization in the 5-ghz band. IEEE Trans Veh Technol 59: 2007-2015

9. Payami S, Tufvesson F (2012) Channel measurements and analysis for very large array systems at $2.6 \mathrm{ghz}$. Eur Conf Antennas Propag, pp: 433-437.

10. Aghaeinezhadfirouzja S, Liu H, Xia B, Tao M (2016) Implementation and measurement of single user mimo testbed for td-lte-a downlink channels. IEEE International Conference on Communication Software and Networks pp: 211-215.

11. Aghaeinezhadfirouzja S, Liu H, Xia B, Luo Q, Guo W (2017) Third dimension for measurement of multi user massive mimo channels based on lte advanced downlink. Signal and Info Process, pp: 758-762.

12. Wu S, Wang CX, Aggoune EHM, Alwakeel MM, He Y (2014) A non-stationary 3- $d$ wideband twin-cluster model for $5 \mathrm{~g}$ massive mimo channels. IEEE J Sel Areas Commun 32: 1207-1218.

13. Wu S, Wang CX, Haas H, Aggoune EHM, Alwakeel MM, Ai B (2015) A non-stationary wideband channel model for massive mimo communication systems. IEEE Trans Wireless Commun 14: 1434-1446.

14. Kammoun A, Khanfir H, Altman Z, Debbah M, Kamoun M (2014) Preliminary results on 3d channel modeling: From theory to standardization. IEEE J Se Areas Commun 32: 1219-1229. 